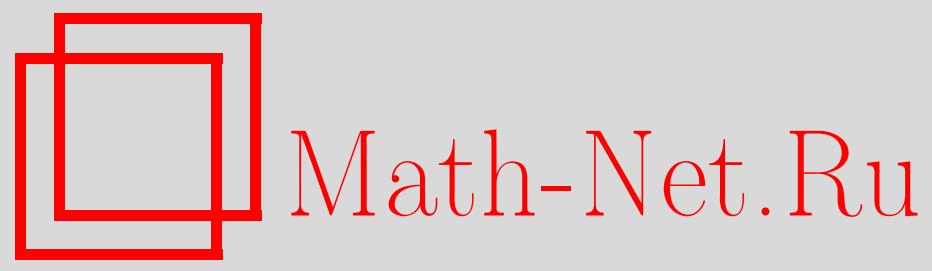

А. Р. Хашимов, О единственности решений второй краевой задачи для уравнения третьего порядка составного типа в неограниченных областях, Вестн. Сам. гос. техн. ун-та. Сер. Физ.-мат. науки, 2012, выпуск 2(), 18-25

DOI: https://doi.org/10.14498/vsgtu912

Использование Общероссийского математического портала Math-Net.Ru подразумевает, что вы прочитали и согласны с пользовательским соглашением

http://www . mathnet.ru/rus/agreement

Параметры загрузки:

IP: 3.91 .87 .62

26 апреля 2023 г., 16:31:07

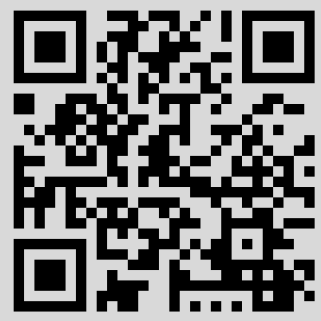


Вестн. Сам. гос. техн. ун-та. Сер. Физ.-мат. науки. 2012. № 2 (27). С. $18-25$

УДК 517.956.6

\title{
О ЕДИНСТВЕННОСТИ РЕШЕНИЙ ВТОРОЙ КРАЕВОЙ ЗАДАЧИ ДЛЯ УРАВНЕНИЯ ТРЕТЬЕГО ПОРЯДКА СОСТАВНОГО ТИПА В НЕОГРАНИЧЕННЫХ ОБЛАСТЯХ
}

\section{A.P. Хашимов}

Ташкентский финансовый институт,

Узбекистан, Ташкент, просп. Амира Темура, 60.

E-mail: khashimov_abdukomil@yahoo.com

\begin{abstract}
Установлены энергетические ощенки типа принципа Сен-Венана для обобщённых решений второй краевой задачи для уравнений третъего порядка составного типа. Использованы методы интегралов энергии и интегральных неравенств. Выявлен широкий класс решений второй краевой задачи в классах функций, растущих на бесконечности, и установлены энергетические оценки решений, позволяюшие исследовать характер стремления к нулю этого решения в окрестности нерегулярных точек границы в зависимости от геометрических характеристик области.
\end{abstract}

Ключевые слова: теорема единственности, принцип Сен-Венана, дифберенииальные уравнения третъего порядка, нерегулярные точки, обобщённые решения, неограниченные области.

Введение. Принцип Сен-Венана в плоской теории упругости выражается в виде априорной оценки для решения бигармонического уравнения, удовлетворяющего на части границы области однородным граничным условиям первой краевой задачи $[1,2]$. Такие энергетические оценки были впервые получены в работах $[3,4]$, но они не учитывают характер изменения формы тела при удалении от той части его границы, где приложены внешние силы. Установлены энергетические оценки типа принципа Сен-Венана, учитывающие характер изменения формы тела, например, для цилиндрического тела [5]. Для системы уравнений теории упругости, в пространственном случае, аналог принципа Сен-Венана, теоремы единственности в неограниченных областях и теоремы типа Фрагмена-Линделёфа приведены в работах [6-9 и др.].

В вышеперечисленных работах объектом исследования являлись уравнения чётного порядка, т.е. уравнения эллиптических и параболических типов. Для уравнений нечётного порядка такого рода исследования проводились в меньших объёмах [10-12]. Так, для уравнения третьего порядка составного типа с граничными условиями первой краевой задачи аналог принципа Сен-Венана и теорема единственности в неограниченных областях получены в работах $[10,11]$. В работе [12] были установлены локальные оценки $W_{2}^{l}$ нормы обобщённых решений уравнения третьего порядка составного типа. Энергетические и локальные оценки вместе дают оценки производных решений любого порядка на бесконечности и в окрестности нерегулярных точек границы.

В настоящей работе обобщаются результаты работ [10-12] и определяется класс единственности решений второй краевой задачи для уравнений третье-

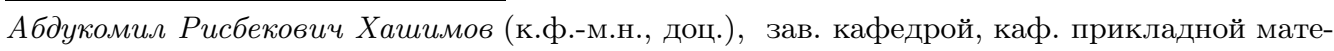
матики. 
го порядка составного типа в классах функций, растущих на бесконечности, а также изучается характер стремления к нулю этого решения в окрестности нерегулярных точек границы в зависимости от геометрических характеристик области. Краевая задача для таких уравнений в ограниченных областях была изучена в работе [13].

Обозначения и постановка задачи. Пусть $\Omega \subset \mathbb{R}_{+}^{n}=\left\{x=\left(x_{1}, x_{2}, \ldots, x_{n}\right)\right.$ : $\left.x_{1}>0\right\}$ - неограниченная область. В области $\Omega$ рассмотрим уравнение

$$
l_{0} A u=f(x)
$$

где $l_{0} u=\alpha^{k} u_{x_{k}}, A u=a^{i j} u_{x_{i} x_{j}}+a^{i} u_{x_{i}}+a u ; \alpha^{k}, a^{i j}, a^{i}, a-$ постоянные.

Относительно оператора $A$ предполагается выполнение условий

$$
a^{i j}=a^{j i}, \quad a_{0}|\xi|^{2} \leqslant a^{i j} \xi_{i} \xi_{j} \leqslant a_{1}|\xi|^{2}, \quad \xi \in \mathbb{R}^{n}
$$

где $a_{0}, a_{1}$ - положительные постоянные.

Обозначим $\nu=\left(\nu_{x_{1}}, \nu_{x_{2}}, \ldots, \nu_{x_{n}}\right)$ - вектор внутренней нормали к границе $\Gamma=\partial \Omega$. Произведем разбиение боковой границы области $\Omega$ :

$\sigma_{1}=\left\{x \in \Gamma: \alpha^{k} \nu_{x_{k}}>0\right\}, \quad \sigma_{2}=\left\{x \in \Gamma: \alpha^{k} \nu_{x_{k}}<0\right\}, \quad \sigma_{0}=\left\{x \in \Gamma: \alpha^{k} \nu_{x_{k}}=0\right\}$.

Для уравнения (1) рассмотрим нижеследующую задачу.

ЗАдАчА. Найти решение уравнения (1), удовлетворяющее краевым условиям

$$
\left.u\right|_{\sigma_{1}}=0,\left.\quad \alpha^{k} u_{x_{k}}\right|_{\Gamma}=0
$$

Введём обозначения $\Omega_{\tau}=\Omega \cap\left\{x: 0<x_{1}<\tau\right\}, \Gamma_{\tau}=\Gamma \cap \partial \Omega_{\tau}, \sigma_{0, \tau}=$ $=\left\{x \in \Gamma_{\tau}: \alpha^{k} \nu_{k}=0\right\}, \sigma_{1, \tau}=\left\{x \in \Gamma_{\tau}: \alpha^{k} \nu_{k}>0\right\}, \sigma_{2, \tau}=\left\{x \in \Gamma_{\tau}: \alpha^{k} \nu_{k}<0\right\}$, $S_{\tau}=\partial \Omega_{\tau} \backslash \Gamma_{\tau}$ и класс функций

$$
H\left(\Omega_{\tau}\right)=\left\{u: l_{0} u \in W_{2}^{1}\left(\Omega_{\tau}\right),\left.l_{0} u\right|_{\Gamma_{\tau}}=0,\left.u\right|_{\sigma_{1, \tau}}=0\right\} .
$$

Отметим, что в работе [13] построено обобщённое решение уравнения (1) в классе $H\left(\Omega_{\tau}\right)$ при $\Gamma_{\tau}=\partial \Omega_{\tau}$.

ОПределЕниЕ. Функцию $u(x)$ будем называть обобщённым решением уравнения (1) в ограниченной области $\Omega_{\tau}$ с граничными условиями $\left.l_{0} u\right|_{\Gamma_{\tau}}=0$ и $\left.u\right|_{\sigma_{1, \tau}}=0$, если $u(x) \in H\left(\Omega_{\tau}\right)$ и удовлетворяет тождеству

$$
p\left(l_{0} u, v\right) \equiv \int_{\Omega_{\tau}}\left[-a^{i j}\left(l_{0} u\right)_{x_{i}} v_{x_{j}}+a^{i}\left(l_{0} u\right)_{x_{i}} v+a\left(l_{0} u\right) v\right] d x=\int_{\Omega_{\tau}} f v d x
$$

для произвольной функции $v \in W_{2}^{1}\left(\Omega_{\tau}\right),\left.v\right|_{\partial \Omega_{\tau}}=0$.

Основные результаты. Установим энергетические оценки типа принципа Сен-Венана, с помощью которых можно доказать теоремы существования и единственности решения краевых задач в классе растущих функций на бесконечности в зависимости от геометрических характеристик области, а также изучить асимптотические свойства решений в окрестности нерегулярных 
точек границы и на бесконечности. Аналогичные исследования для эллиптических и параболических уравнений выполнены многими исследователями (например, см. [6]).

Теорема 1 (АНАЛОГ ПРИНЦИПА Сен-Венана). Пустъ $a \leqslant 0 u f(x)=0$ в $\Omega_{\tau}, \tau \leqslant \tau_{2}$. Если и $(x)$ является обобщённым решением уравнения (1) в $\Omega_{\tau}$ с граничными условиями $\left.l_{0} u\right|_{\Gamma_{\tau}}=\left.0 u u\right|_{\sigma_{1, \tau}}=0$, то для любого $\tau_{1}$ такого, что $0 \leqslant \tau_{1} \leqslant \tau_{2}$, справедлива оченка

$$
\int_{\Omega_{\tau_{1}}} E\left(l_{0} u\right) d x \leqslant \Phi^{-1}\left(\tau_{1}, \tau_{2}\right) \int_{\Omega_{\tau_{2}}} E\left(l_{0} u\right) d x,
$$

где $E\left(l_{0} u\right)=a^{i j}\left(l_{0} u\right)_{x_{i}}\left(l_{0} u\right)_{x_{j}}-a\left(l_{0} u\right)^{2}$. Здесъ $\Phi\left(x_{1}, \tau_{2}\right)$ является решением следующей задачи:

$$
\begin{gathered}
\Phi^{\prime}=-\mu\left(x_{1}\right) \Phi, \quad \tau_{1} \leqslant x \leqslant \tau_{2} \\
\Phi\left(\tau_{2}, \tau_{2}\right)=1
\end{gathered}
$$

$\mu(\tau)$ - любая непрерьвная функиия такая, что

$$
\begin{gathered}
0<\mu(\tau) \leqslant \lambda(\tau) \equiv \inf _{v \in K}\left\{\int_{S_{\tau}} E\left(l_{0} v\right) d x^{\prime}\left|\int_{S_{\tau}} P\left(l_{0} v\right) d x^{\prime}\right|^{-1}\right\}, x^{\prime}=\left(x_{2}, x_{3}, \ldots, x_{n}\right), \\
P\left(l_{0} v\right)=-a^{i 1}\left(l_{0} v\right)_{x_{i}} l_{0} v-a^{1}\left(l_{0} v\right)^{2} / 2
\end{gathered}
$$

$K$-множество дважды непрерывно дифберенцируемых функиий в окрестности $\bar{S}_{\tau}$, удовлетворяющих условию $l_{0} v=0$ на $\bar{S}_{\tau} \cap \Gamma_{\tau}$.

Доказ ательст во. Положим в тождестве $(3) v=u_{m}\left(\psi\left(x_{1}\right)-1\right)$. Здесь $\psi\left(x_{1}\right)=\Phi\left(\tau_{1}, \tau_{2}\right)$, если $0 \leqslant x_{1} \leqslant \tau_{1} ; \psi\left(x_{1}\right)=\Phi\left(x_{1}, \tau_{2}\right)$, если $\tau_{1} \leqslant x_{1} \leqslant \tau_{2} ;$ $\psi\left(x_{1}\right)=1$, если $x_{1} \geqslant \tau_{2} ; u_{m} \in C^{1}\left(\bar{\Omega}_{\tau}\right), \| u_{m}-\left.l_{0} u\right|_{W_{2}^{1}\left(\Omega_{\tau}\right)} \rightarrow 0, l_{0} u \in H\left(\Omega_{\tau}\right)$, $u_{m}=0$ в окрестности $\Gamma_{\tau}$. Тогда $p\left(l_{0} u-u_{m}+u_{m}, u_{m}(\psi-1)\right)=0$ в $\Omega_{\tau_{2}}$. Поэтому

$$
p\left(u_{m}, u_{m}(\psi-1)\right)=\delta_{m} \text { в } \Omega_{\tau_{2}}
$$

где $\delta_{m} \equiv-p\left(l_{0} u-u_{m}, u_{m}(\psi-1)\right)$. Ясно, что $\delta_{m} \rightarrow 0$ при $m \rightarrow \infty$.

Интегрируя (8) по частям, получим

$$
\int_{\Omega_{\tau_{2}}} E\left(u_{m}\right)(\psi-1) d x \leqslant \int_{\Omega_{\tau_{2}}} P\left(u_{m}\right) \psi^{\prime} d x+\delta_{m} .
$$

Отсюда, учитывая условия теоремы, а также соотношения (5) и (7), имеем

$$
\begin{gathered}
\int_{\Omega_{\tau_{2}}} E\left(u_{m}\right)(\psi-1) d x \leqslant \int_{\Omega_{\tau_{2} \backslash \Omega_{\tau_{1}}} P\left(u_{m}\right) \mu \psi d x+\delta_{m}} \\
\int_{\Omega_{\tau_{2}}} E\left(u_{m}\right) \psi d x-\int_{\Omega_{\tau_{2}}} E\left(u_{m}\right) d x \leqslant \int_{\Omega_{\tau_{2}}} E\left(u_{m}\right) \psi d x-\int_{\Omega_{\tau_{1}}} E\left(u_{m}\right) \psi d x+\delta_{m} .
\end{gathered}
$$

Переходя к пределу при $m \rightarrow \infty$ в (9), получим оценку (4). 
Оценим теперь $\lambda\left(x_{1}\right)$ в случае, когда $S_{\tau}$ можно заключить в $(n-1)$-мерный параллелепипед, наименьшее ребро которого равно $\lambda_{1}(\tau)$. Предположим, что $\max \left\{a^{1}, 0\right\}=a_{2}$. Применяя неравенства Фридрихса и Коши-Буняковского, из (7) получаем

$$
\begin{aligned}
& \left|\int_{S_{\tau}} P\left(l_{0} v\right) d x^{\prime}\right| \leqslant\left|\int_{S_{\tau}} a^{i 1} l_{0} v\left(l_{0} v\right)_{x_{i}} d x^{\prime}\right|+\frac{1}{2}\left|\int_{S_{\tau}} a^{1}\left(l_{0} v\right)^{2} d x^{\prime}\right| \leqslant \\
& \leqslant a_{1}\left(\int_{S_{\tau}}\left(l_{0} v\right)^{2} d x^{\prime}\right)^{1 / 2}\left(\int_{S_{\tau}}\left(\left(l_{0} v\right)_{x_{i}}\right)^{2} d x^{\prime}\right)^{1 / 2}+\frac{a_{2} \lambda_{1}^{2}(\tau)}{2 a_{0} \pi^{2}} \int_{S_{\tau}} E\left(l_{0} v\right) d x^{\prime} \leqslant \\
& \leqslant\left(\frac{a_{1} \lambda_{1}(\tau)}{a_{0} \pi}+\frac{a_{2} \lambda_{1}^{2}(\tau)}{2 a_{0} \pi^{2}}\right) \int_{S_{\tau}} E\left(l_{0} v\right) d x^{\prime}
\end{aligned}
$$

Поэтому можно положить

$$
\lambda(\tau)=2 \pi^{2} a_{0}\left(2 \pi a_{1} \lambda_{1}(\tau)+a_{2} \lambda_{1}^{2}(\tau)\right)^{-1} .
$$

Если $a^{1} \leqslant 0$ на $S_{\tau}$, то $a_{2}=0$. Тогда можно положить

$$
\mu(\tau)=\frac{a_{0} \pi}{a_{1} \lambda_{1}(\tau)}
$$

Теперь рассмотрим вопрос построения функции $\Phi\left(x_{1}, \tau_{2}\right)$ в конкретных областях. Для определённости положим $a^{1} \leqslant 0$.

ПримеР 1. Пусть область $\Omega$ при $0 \leqslant \tau_{1} \leqslant x_{1}$ лежит внутри тела вращения $\left|x^{\prime}\right| \leqslant 2^{-1} M\left(x_{1}+1\right)$, т.е. $\lambda_{1}\left(x_{1}\right) \leqslant M\left(x_{1}+1\right), M>0$. Тогда из (10) имеем

$$
\mu(\tau)=\frac{c \pi}{M\left(x_{1}+1\right)}, \quad c=a_{0} / a_{1}
$$

Тогда функция $\Phi\left(x_{1}, \tau_{2}\right)$, которая является решением задачи $(5),(6)$, имеет вид

$$
\Phi\left(x_{1}, \tau_{2}\right)=\left(\frac{\tau_{2}+1}{x_{1}+1}\right)^{m}
$$

где $m=\pi c / M, m>0$. Из неравенства (4) в этом случае получаем, что

$$
\int_{\Omega_{\tau_{1}}} E\left(l_{0} u\right) d x \leqslant \Phi^{-1}\left(\tau_{1}, \tau_{2}\right) \int_{\Omega_{\tau_{2}}} E\left(l_{0} u\right) d x \leqslant\left(\frac{\tau_{1}+1}{\tau_{2}+1}\right)^{m} \int_{\Omega_{\tau_{2}}} E\left(l_{0} u\right) d x .
$$

Полученные точные решение задачи (1), (2) для угловой области показывают, что коэффициент при интеграле в правой части неравенства (11) не может убывать при $\tau_{2} \rightarrow \infty$ быстрее степенной функции.

ПримеР 2. Рассмотрим пример области, для которой

$$
\lambda_{1}\left(x_{1}\right) \leqslant \pi c k^{-1}\left(x_{1}+1\right)^{1-k} .
$$

Это означает, что при $k>1$ область $\Omega$ сужается при $x_{1} \rightarrow \infty$ (т.е. расстояние от $x$ до $\partial \Omega$ при $x_{1} \rightarrow \infty$ стремится к нулю). Если $k=1$, то $\lambda_{1}\left(x_{1}\right) \leqslant \pi c$, и этот 
случай включает области, лежащие в полосе шириной $\pi c$. Если $0<k<1$, то область $\Omega$ может соответственно расширяться при $x_{1} \rightarrow \infty$. Для такой области $\Omega$ можно положить $\mu\left(x_{1}\right)=k\left(x_{1}+1\right)$. Нетрудно проверить, что функция

$$
\Phi\left(x_{1}, \tau_{2}\right)=\exp \left(\left(\tau_{2}+1\right)^{k}-\left(x_{1}+1\right)\right)
$$

удовлетворяет условию (6) и уравнению (5). Поэтому оценка (4) для рассматриваемой области справедлива при

$$
\Phi^{-1}\left(x_{1}, \tau_{2}\right)=\exp \left(-\left(\tau_{2}+1\right)^{k}+\left(x_{1}+1\right)\right) .
$$

Как следствие принципа Сен-Венана получим теорему единственности задачи (1),(2) в неограниченной области $\Omega$ в классах функций, растущих на бесконечности в зависимости от геометрических характеристик области.

Теорема 2. Пусть множество $S_{\tau}=\Omega \cap\left\{x: x_{1}=\tau\right\}$ при любом $\tau>0$ не пусто, $f(x) \equiv 0$ в $\Omega, \alpha^{1} \geqslant 0 u a \leqslant 0$. Пусть и решением уравнения (1) в $\Omega_{\tau}$ с граничными условиями $\left.l_{0} u\right|_{\Gamma_{\tau}}=\left.0 u u\right|_{\sigma_{1, \tau}}=0$ при любом $\tau>0$. Тогда, если для некоторой последовательности $\tau_{m} \rightarrow \infty u$ некоторого положительного постоянного $d_{*}$ имеем

$$
\int_{\Omega_{\tau_{m}}} E\left(l_{0} u\right) d x \leqslant \varepsilon\left(\tau_{m}\right) \Phi\left(d_{*}, \tau_{m}\right)
$$

где $\varepsilon\left(\tau_{m}\right) \rightarrow 0$, при $\tau_{m} \rightarrow \infty$, mо $u(x) \equiv 0$ в $\Omega$.

Прежде чем перейти к доказательству теоремы, приведём следующую лемму [13] без доказательства.

Лемма. Пусть для некоторого $k$ в ограниченной области $\Omega$ выполняется $\left|\alpha^{k}\right| \geqslant \delta_{0}>0$ при $x \in \bar{\Omega} ;$ пересечение множеств $\overline{\sigma_{1}}, \overline{\sigma_{0} \cup \sigma_{2}}$ удовлетворяет условиям конуса. Тогда для любой функиии $\omega$ из пространства $W_{2}^{1}(\Omega)$, где $\left.\omega\right|_{\partial \Omega}=0$, существует решение задачи

$$
l_{0} u=\omega,\left.\quad u_{x_{k}}\right|_{\sigma_{1}}=0
$$

из пространства $W_{2}^{1}(\Omega)$ и для этого решения выполняется оценка

$$
\|u\|_{W_{2}^{1}(\Omega)}=C_{0}\|\omega\|_{W_{2}^{1}(\Omega)}
$$

Теперь, используя эту леммы, докажем теорему 2.

Доказ ательств в. Из (4), учитывая (12), имеем

$$
\int_{\Omega_{d_{*}}} E\left(l_{0} u\right) d x \leqslant \Phi^{-1}\left(d_{*}, \tau_{m}\right) \int_{\Omega_{\tau_{m}}} E\left(l_{0} u\right) d x \leqslant \varepsilon\left(\tau_{m}\right) \rightarrow 0
$$

при $\tau_{m} \rightarrow \infty$. Следовательно, $l_{0} u=0$ в $\Omega_{d_{*}}$. Тогда согласно лемме $u=0$ в $\Omega_{d_{*}}$.

Далее, так как функция $\Phi\left(x_{1}, \tau_{2}\right)$ является решением задачи $(5),(6)$, для любого фиксированного $d_{1}>d_{*}$ имеем

$$
\Phi^{-1}\left(d_{*}, \tau_{m}\right)=C_{1} \Phi^{-1}\left(d_{1}, \tau_{m}\right), \quad C_{1}=\text { const }>0 .
$$


Согласно неравенствам (4) и (12) получаем

$$
\begin{aligned}
\int_{\Omega_{d_{1}}} E\left(l_{0} u\right) d x \leqslant \Phi^{-1}\left(d_{1}, \tau_{m}\right) & \int_{\Omega_{\tau_{m}}} E\left(l_{0} u\right) d x \leqslant \\
& \leqslant \Phi^{-1}\left(d_{1}, \tau_{m}\right) \varepsilon\left(\tau_{m}\right) \Phi\left(d_{*}, \tau_{m}\right)=C_{1}^{-1} \varepsilon\left(\tau_{m}\right) \rightarrow 0
\end{aligned}
$$

при $\tau_{m} \rightarrow \infty$. Следовательно, $l_{0} u=0$ в $\Omega_{d_{1}}$. Тогда согласно лемме $u=0$ в $\Omega_{d_{1}}$. Так как $d_{1}$ выбрано произвольно, то $u=0$ в $\Omega$.

Условие (12) является точным в том смысле, что замена величины $\varepsilon\left(\tau_{m}\right)$ некоторой постоянной $C$ приводит к неединственности решения задачи.

Теперь установим оценки для решения задачи $(1),(2)$, указывающие характер обращения в нуль функции $u(x)$ в окрестности нерегулярной точки $P$ границы области $\Omega$.

ТЕорема 3. Пусть $a \leqslant 0$, область $\Omega$ ограничена и лежит в полуплоскости $\left\{x: x_{1}>0\right\}$, множество $S_{\tau}=\Omega \cap\left\{x: x_{1}=\tau\right\}$ при любом $\tau \in\left(0, \tau^{0}\right)$ не пусто, $\tau^{0}>0, f(x) \equiv 0$ в $\Omega_{\tau^{0}}=\Omega \cap\left\{x_{1}: x_{1}<\tau^{0}\right\}$. Тогда для обобщённого решения и $(x)$ уравнения $(1)$ в области $\Omega_{\tau^{0}}$ с граничными условиями $\left.l_{0} u\right|_{\partial \Omega_{\tau^{0}} \cap \partial \Omega}=0 u$ u $\left.\right|_{\sigma_{1, \tau^{0}}}=0$ справедлива оченка

$$
\begin{aligned}
\int_{\Omega_{\tau^{0}}}\left(l_{0} u\right)^{2} \Lambda\left(x_{1}\right) \Phi\left(x_{1}, \tau^{0}, \varepsilon\right) d x \leqslant & \\
& \leqslant \int_{\Omega_{\tau^{0}}} E\left(l_{0} u\right) \Phi\left(x_{1}, \tau^{0}, \varepsilon\right) d x \leqslant \frac{1}{\varepsilon} \int_{\Omega_{\tau^{0}}} E\left(l_{0} u\right) d x
\end{aligned}
$$

где $\varepsilon=\mathrm{const}>0,0<\varepsilon<1, a \Phi\left(x_{1}, \tau^{0}\right)$ является решением следующей задачи:

$$
\begin{gathered}
\Phi^{\prime}=-(1-\varepsilon) \mu\left(x_{1}\right) \Phi, \quad 0<x<\tau^{0}, \\
\Phi\left(\tau^{0}, \tau^{0}, \varepsilon\right)=1
\end{gathered}
$$

$\mu(\tau)$ - любая непрерьвная функиия такая, что

$$
0<\mu(\tau) \leqslant \lambda(\tau) \equiv \inf _{v \in K}\left\{\int_{S_{\tau}} E\left(l_{0} v\right) d x^{\prime}\left|\int_{S_{\tau}} P\left(l_{0} v\right) d x^{\prime}\right|^{-1}\right\}
$$

где $K$-множество дважды непрерьвно дифферениируемых функиий $v$ в окрестности $\bar{S}_{\tau}$, которые удовлетворяют условию $l_{0} v=0$ на $\bar{S}_{\tau} \cap \Gamma_{\tau}$, а $\Lambda(\tau)$ - непрерывная функиия, удовлетворяющая условию

$$
0<\Lambda(\tau) \leqslant \inf _{v}\left\{\int_{S_{\tau}} E\left(l_{0} v\right) d x^{\prime}\left|\int_{S_{\tau}}\left(l_{0} v\right)^{2} d x^{\prime}\right|^{-1}\right\}
$$

Здесь inf берётся по всем функииям $v$ таким, что $l_{0} v \in C^{1}(\bar{\Omega}), v=0 u$ $l_{0} v=0$ в $\sigma_{1}$ и $\partial \Omega$ соответственно.

Доказательство. Положим в $(3) v(x)=u_{m}\left(\psi\left(x_{1}, \delta\right)-1\right)$, где $\psi\left(x_{1}, \delta\right)=$ $=\Phi\left(\delta, \tau^{0}, \varepsilon\right)$, если $0 \leqslant x_{1} \leqslant \delta ; \psi\left(x_{1}, \delta\right)=\Phi\left(x_{1}, \tau^{0}, \varepsilon\right)$, если $0<\delta \leqslant x_{1} \leqslant \tau^{0}$; 
$\psi\left(x_{1}, \delta\right)=1$, если $x_{1} \geqslant \tau^{0} ; u_{m} \in C^{1}\left(\overline{\Omega_{\tau^{0}}}\right) ; \| u_{m}-\left.l_{0} u\right|_{W_{2}^{1}\left(\Omega_{\tau^{0}}\right)} \rightarrow 0, l_{0} u \in H\left(\Omega_{\tau^{0}}\right)$, $u_{m}=0$ в окрестности $\Gamma=\partial \Omega_{\tau^{0}}$. Тогда

$$
p\left(l_{0} u-u_{m}+u_{m}, u_{m}(\psi-1)\right)=0 \text { в } \Omega_{\tau^{0}} .
$$

Поэтому

$$
p\left(u_{m}, u_{m}(\psi-1)\right)=\delta_{m} \quad \text { в } \Omega_{\tau^{0}}
$$

где $\delta_{m} \equiv-p\left(l_{0} u-u_{m}, u_{m}(\psi-1)\right)$, и ясно, что $\delta_{m} \rightarrow 0$, при $m \rightarrow \infty$.

Интегрируя (17) по частям, получим

$$
\int_{\Omega_{\tau^{0}}} E\left(u_{m}\right)(\psi-1) d x \leqslant \int_{\Omega_{\tau^{0}}} P\left(u_{m}\right) \psi^{\prime} d x+\delta_{m} .
$$

Отсюда, учитывая условия теоремы, а также соотношения (14), (15) и (16), получим

$$
\begin{aligned}
& \Phi\left(\delta, \tau^{0}, \varepsilon\right) \int_{\Omega_{\delta}} E\left(u_{m}\right) d x+\int_{\Omega_{\tau^{0}} \backslash \Omega_{\delta}} E\left(u_{m}\right) \Phi\left(x_{1}, \tau^{0}, \varepsilon\right) d x \leqslant \\
& \quad \leqslant \int_{\Omega_{\tau^{0}}} E\left(u_{m}\right) d x+(1-\varepsilon) \int_{\Omega_{\tau^{0} \backslash \Omega_{\delta}}} E\left(u_{m}\right) \Phi\left(x_{1}, \tau^{0}, \varepsilon\right) d x+\delta_{m} .
\end{aligned}
$$

Неравенство в (13) вытекает из (18) при предельном переходе $m \rightarrow \infty$ и $\delta \rightarrow 0$.

\section{БИБЛИОГРАФИЧЕСКИЙ СПИСОК}

1. Saint-Venant A. J. C. B. Memoire sur la Torsion des Prismes // Mem. Divers Savants, 1855. Vol. 14,. Pp. 233-560.

2. Gurtin M.E. The Linear Theory of Elasticity/ In: Handbuch der Physik. Vol. VIa/2. Heidelberg: Springer-Verlag, 1972. Pp. 1-296.

3. Knowles J.K. On Saint-Venant's principle in the two-dimensional linear theory of elasticity // Arch. Ration. Mech. Anal., 1966. Vol.21, no. 1. Pp. 1-22.

4. Flavin J.N. On Knowles' version of Saint-Venant's Principle in two-dimensional elastostatics // Arch. Ration. Mech. Anal., 1974. Vol. 53, no. 4. Pp. 366-375.

5. Toupin R. A. Saint-Venant's Principle// Arch. Ration. Mech. Anal., 1965. Vol.18, no. 2. Pp. 83-96.

6. Oleinik O.A., Iosifian G.A. On singularities at the boundary points and uniqueness theorems for solutions of the first boundary value problem of elasticity // Comm. Part. Differ. Equat., 1977. Vol. 2, no. 9. Pp. 937-969.

7. Ковалевский А.А., Скрыпник И.И., Шишков А.Е. Сингулярные решения нелинейных эллиптических и параболических уравнений. Киев: Наукова думка, 2010. 499 с. [Kovalevskiy A. A., Skrypnik I. I., Shishkov A. E. Singular solutions of nonlinear elliptic and parabolic equations. Kiev: Naukova Dumka, 2010. 499 pp.]

8. Galaktionov V. A., Shishkov A. E. Self-similar boundary blow-up for higher-order quasilinear parabolic equations // Proc. R. Soc. Edinb. A. Vol. 135, no.6. Pp. 1195-1227(33).

9. Шишков A.E. Классы единственности обобщенных решений краевых задач для параболических уравнений в неограниченных цилиндрических областях // Дифферени. уравнения, 1990. Т. 26, № 9. С. 1627-1633; англ. пер.: Shishkov A. E. Uniqueness classes of generalized solutions of boundary value problems for parabolic equations in unbounded noncylindrical domains // Differ. Equat., 1990. Vol. 26, no. 9. Pp. 1212-1218. 
10. Джураев Т.Д., Хашимов А.Р. О существовании решений первой краевой задачи для уравнений третьего порядка составного типа в неограниченной области $/ / B e c m \mu$. Сам. гос. техн. ун-та. Сер. Физ.-мат. науки, 2003. №19. С. 5-7. [Dzhuraev T.D., Khashimov A.R. On the existence of value boundary problem first solutions for equations of third order compound type in an unbounded region // Vestn. Samar. Gos. Tekhn. Univ. Ser. Fiz.-Mat. Nauki, 2003. no. 19. Pp. 5-7].

11. Хашимов A.P. О единственности решения одной краевой задачи для общего линейного уравнения третьего порядка составного типа в неограниченных областях // Узбек. мam. ж., 1999. №3. C. 77-85. [Khashimov A. R. On the uniqueness of the solution of a boundary value problem for a general third-order equation of composite type in unbounded domains // Uzbek. Mat. Zh., 1999. no. 3. Pp. 77-85].

12. Хашимов A.P. О локальных оценках обобщённых решений уравнений третьего порядка составного типа / В сб.: Неклассический уравнений математической физики: Международный семинар, посвящённый 60-летию со дня рождения профессора В. Н. Врагова (Новосибирск, 3-5 октября 2005 г.). Новосибирск, 2005. С. 285-291. [Khashimov A. R. On local estimations of generalized solutions to third order composite type equations / In: Nonclassical Equations of Mathematical Physics. Novosibirsk, 2005. Pp. 285-291].

13. Кожанов А.И. Краевые задачи для уравнений математической физики нечетного порядка. Новосибирск: Новосибирск. гос. унив., 1990. 132 с. [Boundary value problems for odd-order equations of mathematical physics. Novosibirsk: Novosibirsk. Gos. Univ., 1990. 132 pp.]

Поступила в редакцию 09/I/2011;

в окончательном варианте - 04/03/2012.

MSC: 35A02; 35B40, 35B45, 35D30, 35G15

\section{ON UNIQUENESS OF THE SECOND BOUNDARY VALUE PROBLEM SOLUTIONS FOR THE THIRD ORDER COMPOSITE TYPE EQUATION IN UNBOUNDED DOMAINS}

\section{A. R. Khashimov}

Tashkent Financial Institute,

60, Amir Temur Prospekt, Tashkent, Uzbekistan.

E-mail: khashimov_abdukomil@yahoo.com

In the paper the second boundary value problem for the third order composite type equations is investigated. We established Saint-Venant's type energy estimates for weak solutions of the problem on Sobolev classes. The obtained estimates are used to prove uniqueness theorems in the classes of functions growing at infinity. These uniqueness classes depend on the geometrical characteristics of the domain. Moreover, energy estimates allowing us to investigate behavior of solution in the neighborhood of singular points were obtained.

Key words: uniqueness theorem, Saint-Venant's principle, third order differential equations, singular points, general solutions, unbounded domains.

Original article submitted 09/I/2011;

revision submitted $04 / 03 / 2012$.

Abdukomil R. Khashimov (Ph.D. (Phys. \& Math.)), Head of Dept., Dept. of Applied Mathematics. 